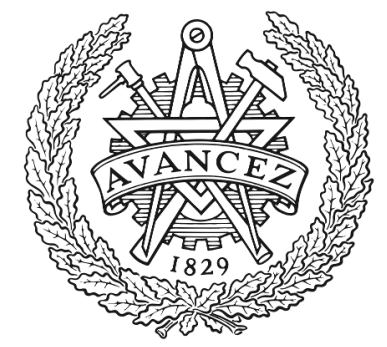

CHALMERS

UNIVERSITY OF TECHNOLOGY

\title{
Performance of location and orientation estimation in 5G mmWave systems: Uplink vs downlink
}

Downloaded from: https://research.chalmers.se, 2023-04-26 07:02 UTC

Citation for the original published paper (version of record):

Abu-Shaban, Z., Zhou, X., Abhayapala, T. et al (2018). Performance of location and orientation estimation in 5G mmWave systems: Uplink vs downlink. IEEE Wireless Communications and Networking Conference, WCNC, 2018-April: 1-6. http://dx.doi.org/10.1109/WCNC.2018.8376990

N.B. When citing this work, cite the original published paper. 


\title{
Performance of Location and Orientation Estimation in 5G mmWave Systems: Uplink vs Downlink
}

\author{
Zohair Abu-Shaban*, Xiangyun Zhou*, Thushara Abhayapala*, Gonzalo Seco-Granados ${ }^{\dagger}$, Henk Wymeersch ${ }^{\ddagger}$ \\ *The Australian National University, Australia. Email: \{zohair.abushaban, xiangyun.zhou, thushara.abhayapala\}@anu.edu.au \\ †Universitat Autònoma de Barcelona, Spain. Email: gonzalo.seco@uab.es \\ ‡Chalmers University of Technology, Sweden. Email: henkw@chalmers.se
}

\begin{abstract}
The fifth generation of mobile communications (5G) is expected to exploit the concept of location-aware communication systems. Therefore, there is a need to understand the localization limits in these networks, particularly, using millimeterwave technology (mmWave). Contributing to this understanding, we consider single-anchor localization limits in terms of 3D position and orientation error bounds for mmWave multipath channels, for both the uplink and downlink. It is found that uplink localization is sensitive to the orientation angle of the user equipment (UE), whereas downlink is not. Moreover, in the considered outdoor scenarios, reflected and scattered paths generally improve localization. Finally, using detailed numerical simulations, we show that mmWave systems are in theory capable of localizing a UE with sub-meter position error, and sub-degree orientation error.
\end{abstract}

\section{INTRODUCTION}

In the recent years, millimeter-wave (mmWave) technology has received a considerable attention as a candidate technology for the fifth generation of mobile communication (5G). MmWave carrier frequencies range between 30 and $300 \mathrm{GHz}$. Having tiny wavelengths allows packing hundreds of antennas in a small area, making mmWave massive MIMO an attractive technology for 5G. Location-aware communication systems are expected to have various applications in 5G [1], such as vehicular communications [2], assisted living applications [3], or to support the communication robustness and effectiveness in different aspects such as resource allocation [4], beamforming [5], and pilot assignment [6]. This makes the study of performance bounds on the user equipment (UE) location and orientation a priority. The orientation importance stems from the application of directional beamforming whose coverage depends, among others, on where the beams are pointed.

Although the position information could be obtained through the time-based GPS, it degrades indoors and in urban canyons and cannot directly provide orientation. To overcome these shortcomings, research has been directed towards alternative spatio-temporal radio localization techniques. To understand their fundamental behavior, the Cramér-Rao lower bound (CRLB) [7] or related bounds can be used. The square-root of the CRLB of the position and the orientation are termed the position error bound (PEB), and the orientation error bound (OEB), respectively. PEB and OEB can be computed indirectly by transforming the Fisher information matrix (FIM) of the channel parameters, namely: directions of arrival (DOA), directions of departure (DOD), and time of arrival (TOA), as in

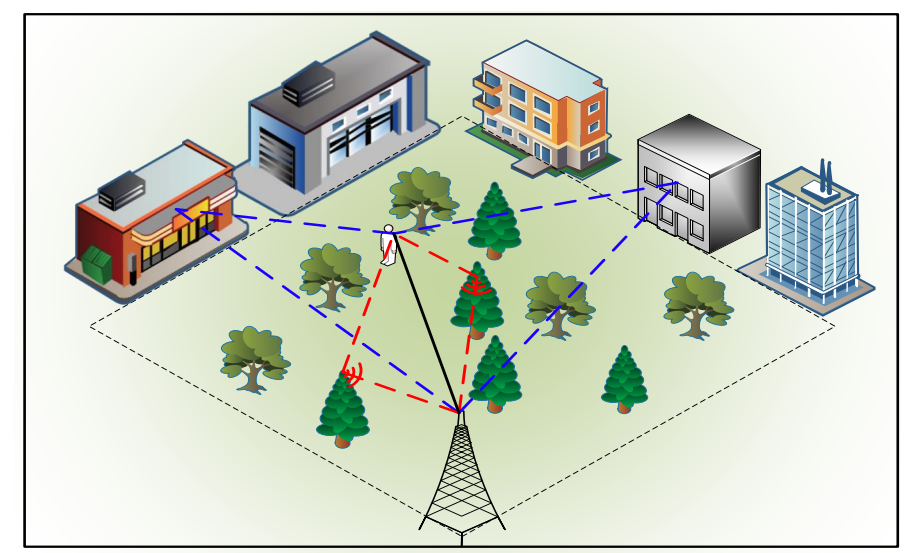

Fig. 1. A single anchor $5 \mathrm{G}$ localization scenario with LOS (black), 2 reflectors (blue) and 2 scatterers (red). The objective is to determine location and orientation of the user.

[8], [9] that considered 2D cooperative wideband localization, highlighting the benefit of large bandwidths.

MmWave massive MIMO benefits from large antenna arrays and large bandwidths. Therefore, mmWave localization is very promising. The PEB and OEB for 2D mmWave downlink localization using uniform linear arrays are reported in [10], while 2D uplink multi-anchor localization is considered in [11]. Moreover, for indoor scenarios, the PEB and OEB are investigated in [12] for 3D mmWave uplink localization with a single beam whose direction is assumed to be known. Although multipath environments are considered in [10]-[12], the difference between the uplink and downlink for 3D and 2D with large number of antennas and analog transmit beamforming, and the effect of reflectors and scatterers on the localization performance have not been analyzed.

In this paper, we address these two issues and study the uplink and downlink PEB and OEB under multipath propagation for 3D mmWave single-anchor localization. We use directional beamforming and antenna arrays with arbitrary but known geometries. In addition, we highlight the effect of scatterers and reflectors on both of these bounds, and give a more visual illustration of the scenarios studied (see Fig. 1). We derive these bounds by transforming the FIM of the channel parameters into a FIM of position and orientation. These results are part of a detailed study that can be found in [13]. 


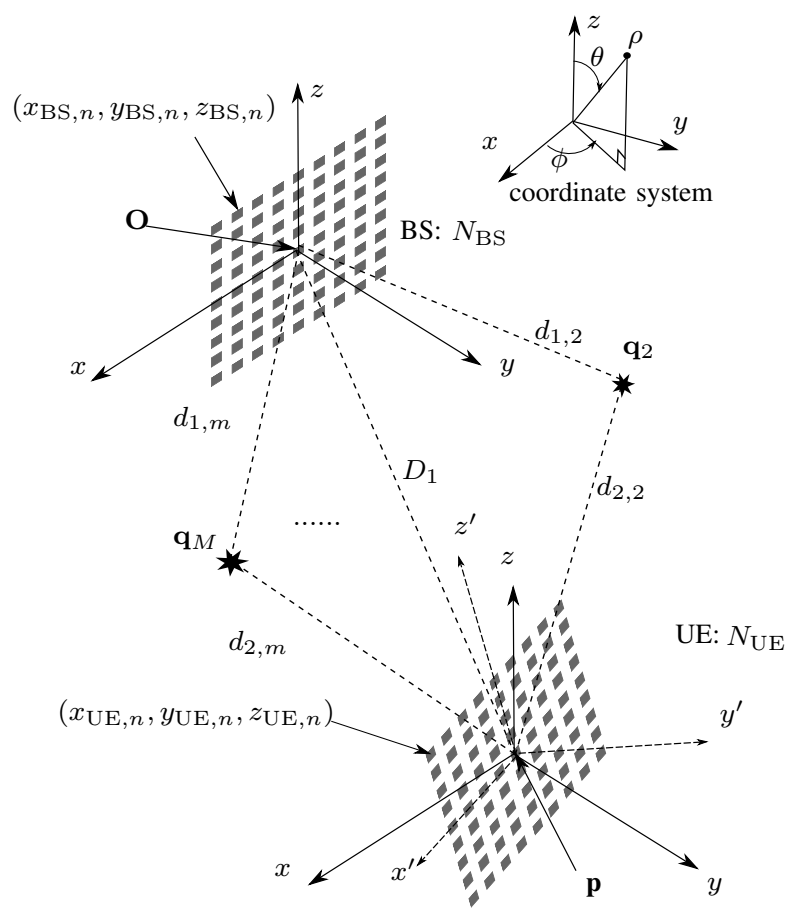

Fig. 2. A URA of $N_{\mathrm{UE}}=N_{\mathrm{BS}}=81$ antennas, and $M$ paths. We use the spherical coordinate system highlighted in the top right corner. The axes rotated by orientation angles $\left(\theta_{0}, \phi_{0}\right)$ are labeled $x^{\prime}, y^{\prime}, z^{\prime}$.

\section{System Model}

\section{A. System Geometry}

Consider a BS equipped with an array of $N_{\mathrm{BS}}$ antennas whose centroid is located at the origin $(\mathbf{O})$ and orientation is $\mathbf{o}_{\mathrm{BS}}=[0,0]^{\mathrm{T}}$. On the other hand, the UE, equipped with a second array of $N_{\mathrm{UE}}$ antennas, has a centroid located at unknown position $\mathbf{p}=\left[p_{x}, p_{y}, p_{z}\right]^{\mathrm{T}}$ and orientation $\mathbf{o}=\left[\theta_{0}, \phi_{0}\right]^{\mathrm{T}}$. Both arrays are arranged in an arbitrary but known geometries. Fig. 2 illustrates a uniform rectangular array (URA) as an example array. The channel comprises $M \geq 1$ paths, where the first path is LOS, while the other $M-1$ paths are associated with clusters located at $\mathbf{q}_{m}=\left[q_{m, x}, q_{m, y}, q_{m, z}\right]^{\mathrm{T}}, 2 \leq m \leq M$. These clusters can be either reflectors or scatterers. We assume that $M$ is estimated a priori using a compressive sensing-based method [10] or a correlation-based method [14]. In mmWave propagation, $M \ll \min \left(N_{\mathrm{R}}, N_{\mathrm{T}}\right)$ [15] and corresponds to single-bounce reflections [10]. Consequently, the channel can be considered spatially sparse and the parameters of different paths are assumed to be distinct, i.e., we assume unique DOAs, DODs and TOA.

\section{B. Channel Model}

Denote by $\left(\theta_{\mathrm{T}, m}, \phi_{\mathrm{T}, m}\right)$ and $\left(\theta_{\mathrm{R}, m}, \phi_{\mathrm{R}, m}\right), 1 \leq m \leq M$, the $m^{\text {th }}$ DOD and DOA, respectively. Then, the related unitnorm array response vectors are given by

$$
\begin{aligned}
& \mathbf{a}_{\mathrm{T}, m}\left(\theta_{\mathrm{T}, m}, \phi_{\mathrm{T}, m}\right) \triangleq \frac{1}{\sqrt{N_{\mathrm{T}}}} e^{-j \boldsymbol{\Delta}_{\mathrm{T}}^{\mathrm{T}} \mathbf{k}\left(\theta_{\mathrm{T}, m}, \phi_{\mathrm{T}, m}\right)}, \in \mathbb{C}^{N_{\mathrm{R}}} \\
& \mathbf{a}_{\mathrm{R}, m}\left(\theta_{\mathrm{R}, m}, \phi_{\mathrm{R}, m}\right) \triangleq \frac{1}{\sqrt{N_{\mathrm{R}}}} e^{-j \boldsymbol{\Delta}_{\mathrm{R}}^{\mathrm{T}} \mathbf{k}\left(\theta_{\mathrm{R}, m}, \phi_{\mathrm{R}, m}\right)}, \in \mathbb{C}^{N_{\mathrm{T}}}
\end{aligned}
$$

where $\mathbf{k}(\theta, \phi)=\frac{2 \pi}{\lambda}[\cos \phi \sin \theta, \sin \phi \sin \theta, \cos \theta]^{\mathrm{T}}$ is the wavenumber vector, $\lambda$ is the wavelength,
$\boldsymbol{\Delta}_{\mathrm{R}} \triangleq\left[\mathbf{u}_{\mathrm{R}, 1}, \mathbf{u}_{\mathrm{R}, 2}, \cdots, \mathbf{u}_{\mathrm{R}, N_{\mathrm{R}}}\right], \mathbf{u}_{\mathrm{R}, n} \triangleq\left[x_{\mathrm{R}, n}, y_{\mathrm{R}, n}, z_{\mathrm{R}, n}\right]^{\mathrm{T}}$ is a vector of Cartesian coordinates of the $n^{\text {th }}$ receiver element, and $N_{\mathrm{R}}$ is the number of receiving antennas. $N_{\mathrm{T}}, \boldsymbol{\Delta}_{\mathrm{T}}$ and $\mathbf{u}_{\mathrm{T}, n}$ are defined similarly. The angle parameters are dropped from the notation of $\mathbf{a}_{\mathrm{T}, m}$, and $\mathbf{a}_{\mathrm{R}, m}$ hereafter.

Denoting the TOA of the $m^{\text {th }}$ path by $\tau_{m}$, the channel can be expressed ${ }^{1}$ as

$$
\begin{aligned}
\mathbf{H}(t) & =\sum_{m=1}^{M} \mathbf{H}_{m} \delta\left(t-\tau_{m}\right), \\
\mathbf{H}_{m} & \triangleq \sqrt{N_{\mathrm{R}} N_{\mathrm{T}}} \beta_{m} \mathbf{a}_{\mathrm{R}, m} \mathbf{a}_{\mathrm{T}, m}^{\mathrm{H}} \in \mathbb{C}^{N_{\mathrm{R}} \times N_{\mathrm{T}}},
\end{aligned}
$$

where, from Fig. 2, $\tau_{m}=D_{m} / c$, and $D_{m}=d_{1, m}+d_{2, m}$, for $m>1$ and $\beta_{m}$ is the complex gain of the $m^{\text {th }}$ path.

\section{Transmission and Reception Model}

The transmitted signal is modeled by $\sqrt{E_{\mathrm{s}}} \mathbf{F} \mathbf{s}(t)$, where $E_{\mathrm{s}}$ is the transmitted energy per symbol duration, $\mathbf{F} \triangleq$ $\left[\mathbf{f}_{1}, \mathbf{f}_{2}, \ldots \mathbf{f}_{N_{\mathrm{B}}}\right]$ is a directional beamforming matrix, such that

$$
\mathbf{f}_{\ell}=\frac{1}{\sqrt{N_{\mathrm{B}}}} \mathbf{a}_{\mathrm{T}, \ell}\left(\theta_{\ell}, \phi_{\ell}\right), \quad 1 \leq \ell \leq N_{\mathrm{B}}
$$

is a beam pointing towards $\left(\theta_{\ell}, \phi_{\ell}\right)$ of the same form as (1), and $N_{\mathrm{B}}$ is the number of transmitted beams. The pilot signal $\mathbf{s}(t) \triangleq\left[s_{1}(t), s_{2}(t), \ldots, s_{N_{\mathrm{B}}}(t)\right]^{\mathrm{T}}$ is expressed as

$$
s_{\ell}(t)=\sum_{k=0}^{N_{\mathrm{s}}-1} a_{\ell, k} p\left(t-k T_{\mathrm{s}}\right), 1 \leq \ell \leq N_{\mathrm{B}},
$$

where $a_{\ell, k}$, for each $\ell$, is a sequence of known unit-energy pilot symbols transmitted over the $\ell^{\text {th }}$ beam. $p(t)$ is a unitenergy pulse with a power spectral density (PSD), denoted by $|P(f)|^{2}$. In (6), $N_{\mathrm{s}}$ is the number of pilot symbols and $T_{\mathrm{s}}$ is the symbol duration, leading to a total observation time of $T_{\mathrm{O}} \approx N_{\mathrm{s}} T_{\mathrm{s}}$. To keep the transmitted power fixed with $N_{\mathrm{T}}$, we set $\operatorname{Tr}\left(\mathbf{F}^{\mathrm{H}} \mathbf{F}\right)=1, \mathbf{s}(t) \mathbf{s}^{\mathrm{H}}(t)=\mathbf{I}_{N_{\mathrm{B}}}$, where $\operatorname{Tr}(\cdot)$ denotes the matrix trace, and $\mathbf{I}_{N_{\mathrm{B}}}$ is the $N_{\mathrm{B}}$-dimensional identity matrix. Although the sequences may be separated spatially by orthogonal beams, having orthogonal sequences facilitates DOD estimation be relating given sequence to a given beam.

The received signal observed at the input of the receive beamformer is given by

$$
\mathbf{r}(t) \triangleq \sum_{m=1}^{M} \sqrt{E_{\mathrm{s}}} \mathbf{H}_{m} \mathbf{F s}\left(t-\tau_{m}\right)+\mathbf{n}(t), \quad t \in\left[0, T_{\mathrm{o}}\right],
$$

where $\mathbf{n}(t) \triangleq\left[n_{1}(t), n_{2}(t), \ldots, n_{N_{\mathrm{T}}}(t)\right]^{\mathrm{T}} \in \mathbb{C}^{N_{\mathrm{R}}}$ is zero-mean white Gaussian noise (AWGN) with PSD $N_{0}$.

\section{3D Single-User Localization Problem}

Our objective is to derive the UE PEB and OEB, based on the observed signal, $\mathbf{r}(t)$, for both the uplink and downlink. We achieve this in two steps: firstly, we derive the FIM of the channel parameters: directions of arrival, $\left(\theta_{\mathrm{R}, m}, \phi_{\mathrm{R}, m}\right)$, directions of departure, $\left(\theta_{\mathrm{T}, m}, \phi_{\mathrm{T}, m}\right)$, times of arrival $\tau_{m}$, and paths gains, $\beta_{m} \forall 1 \leq m \leq M$. Secondly, we transform this FIM into the position domain.

\footnotetext{
${ }^{1}$ We use a narrow-band array model, so that $A_{\max } \ll c / W$, where $A_{\max }$ is maximum array aperture, $c$ is speed of light, and $W$ is the bandwidth.
} 


\section{Fisher Information Matrix of The Channel PARAMETERS}

To derive the FIM of channel parameters, let us define the following parameter vector

$$
\varphi \triangleq\left[\boldsymbol{\varphi}_{1}^{\mathrm{T}}, \boldsymbol{\varphi}_{2}^{\mathrm{T}}, \cdots, \boldsymbol{\varphi}_{M}^{\mathrm{T}}\right]^{\mathrm{T}},
$$

where $\varphi_{m}^{\mathrm{T}} \triangleq\left[\theta_{\mathrm{R}, m}, \phi_{\mathrm{R}, m}, \theta_{\mathrm{T}, m}, \phi_{\mathrm{R}, m}, \tau_{m}, \beta_{\mathrm{R}, m}, \beta_{\mathrm{I}, m},\right]$, $\beta_{\mathrm{R}, m} \triangleq \Re\left\{\beta_{m}\right\}$, and $\beta_{\mathrm{I}, m} \triangleq \Im\left\{\beta_{m}\right\}$ are the real and imaginary parts of $\beta_{m}$, respectively. Denote the $u^{\text {th }}$ element in $\varphi$ by $\varphi_{u}$. Then, the corresponding FIM, partitioned into FIMs of the individual paths is structured as

$$
\mathbf{J}_{\varphi} \triangleq\left[\begin{array}{ccc}
\mathbf{J}_{\varphi_{1} \varphi_{1}} & \cdots & \mathbf{J}_{\varphi_{1} \varphi_{M}} \\
\vdots & \ddots & \vdots \\
\mathbf{J}_{\varphi_{1} \varphi_{M}} & \cdots & \mathbf{J}_{\varphi_{M} \varphi_{M}}
\end{array}\right] .
$$

Since $\mathbf{n}(t)$ is AWGN, from [7],

$$
\begin{aligned}
{\left[\mathbf{J}_{\varphi}\right]_{u, v} \triangleq \frac{1}{N_{0}} \int_{0}^{T_{\mathrm{o}}} \Re\left\{\frac{\partial \boldsymbol{\mu}_{\varphi}^{\mathrm{H}}(t)}{\partial \varphi_{u}} \frac{\partial \boldsymbol{\mu}_{\varphi}(t)}{\partial \varphi_{v}}\right\} \mathrm{d} t, } \\
\boldsymbol{\mu}_{\varphi}(t) \triangleq \sqrt{N_{\mathrm{R}} N_{\mathrm{T}} E_{\mathrm{s}}} \sum_{m=1}^{M} \beta_{m} \mathbf{a}_{\mathrm{R}, m} \mathbf{a}_{\mathrm{T}, m}^{\mathrm{H}} \mathbf{F} \mathbf{s}\left(t-\tau_{m}\right) .
\end{aligned}
$$

MmWave systems enjoy a spatially sparse channel, employ a larger number of antennas at the transmitter and receiver, and utilize a large bandwidth. Taking these particularities into account, it was shown in [13] that $\mathbf{J}_{\boldsymbol{\varphi}_{m} \boldsymbol{\varphi}_{m^{\prime}}} \approx \mathbf{0}, \forall m \neq m^{\prime}$. In other words, $\mathbf{J}_{\varphi}$ can be considered a block-diagonal matrix, and the paths are almost orthogonal, and carry independent information.

\section{Fisher INFORMATION OF THE LOCATION PARAMETERS: UPLINK VS. DOWNLINK}

In this section, we derive the PEB and OEB by transforming the FIM of the multipath channel parameters in (9), into an FIM of orientation, position, and clusters location [7]. First, we start by a general derivation of these bounds, before we highlight how the transmission being uplink or downlink impacts them.

\section{A. General Derivation}

Define $\varphi_{\mathrm{L}} \triangleq\left[\mathbf{o}^{\mathrm{T}}, \mathbf{p}^{\mathrm{T}}, \mathbf{q}^{\mathrm{T}}\right]$ and $\mathbf{q} \triangleq\left[\mathbf{q}_{2}^{\mathrm{T}}, \mathbf{q}_{3}^{\mathrm{T}}, \cdots, \mathbf{q}_{M}^{\mathrm{T}}\right]^{\mathrm{T}}$. Then, the transformation is given by

$$
\mathbf{J}_{\varphi_{\mathrm{L}}} \triangleq \mathbf{T} \mathbf{J}_{\varphi} \mathbf{T}^{\mathrm{T}}
$$

where

$$
\mathbf{T} \triangleq\left[\mathbf{T}_{1}, \mathbf{T}_{2}, \cdots, \mathbf{T}_{M}\right]=\left[\begin{array}{llll}
\frac{\partial \varphi_{1}^{\mathrm{T}}}{\partial \mathbf{o}^{\mathrm{T}}} & \frac{\partial \boldsymbol{\varphi}_{2}^{\mathrm{T}}}{\partial \mathbf{0}_{\mathrm{T}}} & \ldots & \frac{\partial \boldsymbol{\varphi}_{M}^{\mathrm{T}}}{\partial \mathbf{Q}^{\mathrm{T}}} \\
\frac{\partial \boldsymbol{\varphi}_{1}^{\mathrm{T}}}{\partial \mathbf{p}_{\mathrm{T}}^{\mathrm{T}}} & \frac{\partial \boldsymbol{\varphi}_{2}^{\mathrm{T}}}{\partial \mathbf{p}_{\mathrm{T}}^{\mathrm{T}}} & \ldots & \frac{\partial \boldsymbol{\varphi}_{M}^{\mathrm{T}}}{\partial \mathbf{p}_{\mathrm{T}}} \\
\frac{\partial \boldsymbol{\varphi}_{1}^{\mathrm{T}}}{\partial \mathbf{q}} & \frac{\partial \boldsymbol{\varphi}_{2}^{\mathrm{T}}}{\partial \mathbf{q}} & \ldots & \frac{\partial \boldsymbol{\varphi}_{M}^{\mathrm{T}}}{\partial \mathbf{q}}
\end{array}\right]
$$

Partitioning $\mathbf{J}_{\varphi_{\mathrm{L}}}$ into

$$
\mathbf{J}_{\varphi_{\mathrm{L}}}=\left[\begin{array}{cc}
\mathbf{J}_{\mathbf{o}, \mathbf{p}} & \mathbf{\Phi} \\
\boldsymbol{\Phi}^{T} & \mathbf{J}_{\mathbf{q}}
\end{array}\right]
$$
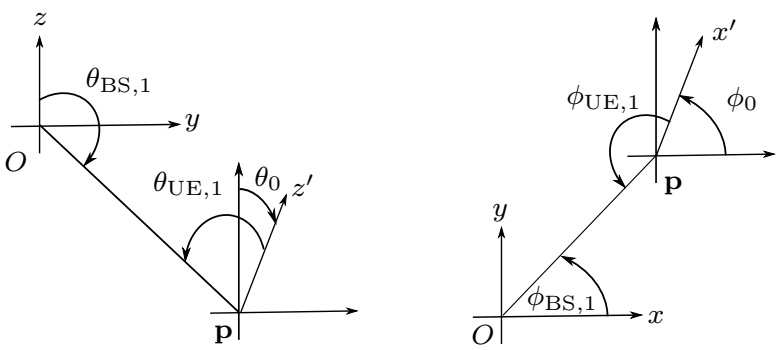

Fig. 3. LOS relationship between orientation angles and the angles of BS and $\mathrm{UE}$, in the elevation (left), and azimuth (right).

where $\mathbf{J}_{\mathbf{o}, \mathbf{p}} \in \mathbb{R}^{5 \times 5}, \mathbf{J}_{\mathbf{q}} \in \mathbb{R}^{3(M-1) \times 3(M-1)}$, we can write the equivalent EFIM of $\mathbf{p}$ and $\mathbf{o}$ using Schur's complement as

$$
\mathbf{J}_{\mathbf{o}, \mathbf{p}}^{\mathrm{e}}=\mathbf{J}_{\mathbf{o}, \mathbf{p}}-\boldsymbol{\Phi} \mathbf{J}_{\mathbf{q}}^{-1} \boldsymbol{\Phi}^{\mathrm{T}} \text {. }
$$

Finally, the PEB and OEB are given by [8]

$$
\begin{aligned}
\mathrm{OEB} & =\sqrt{\left[\left(\mathbf{J}_{\mathbf{o}, \mathbf{p}}^{\mathbf{e}}\right)^{-1}\right]_{1,1}+\left[\left(\mathbf{J}_{\mathbf{o}, \mathbf{p}}^{\mathbf{e}}\right)^{-1}\right]_{2,2}}, \\
\mathrm{PEB} & =\sqrt{\left[\left(\mathbf{J}_{\mathbf{o}, \mathbf{p}}^{\mathbf{e}}\right)^{-1}\right]_{3,3}+\left[\left(\mathbf{J}_{\mathbf{o}, \mathbf{p}}^{\mathbf{e}}\right)^{-1}\right]_{4,4}+\left[\left(\mathbf{J}_{\mathbf{o}, \mathbf{p}}^{\mathbf{e}}\right)^{-1}\right]_{5,5}} .
\end{aligned}
$$

\section{B. Parameter Transformation for Uplink and Downlink}

The relationships governing the UE position and orientation with the DODs and DOAs are different. Therefore, unlike $\mathbf{J}_{\varphi}$, the structure of $\mathbf{T}$ and, effectively, $\mathbf{J}_{\mathbf{o}, \mathbf{p}}^{\mathbf{e}}$, depends on whether the localization is performed in the uplink or in the downlink. Thus, we switch to the explicit notation with the subscripts $\mathrm{BS}$ and UE, respectively, replacing the $\mathrm{R}$ and $\mathrm{T}$ in the uplink expressions, and $\mathrm{T}$ and $\mathrm{R}$ in the downlink expressions.

With the BS located at the origin, the geometry relating the LOS angle parameters is illustrated in Fig. 3, from which we can derive the following equations

$$
\begin{aligned}
\theta_{\mathrm{UE}, 1} & =\theta_{0}-\tan ^{-1}\left(p_{y} / p_{z}\right)+\pi, \\
\phi_{\mathrm{UE}, 1} & =-\phi_{0}+\tan ^{-1}\left(p_{y} / p_{x}\right)+\pi, \\
\theta_{\mathrm{BS}, 1} & =\tan ^{-1}\left(p_{y} / p_{z}\right), \\
\phi_{\mathrm{BS}, 1} & =\tan ^{-1}\left(p_{y} / p_{x}\right), \\
\tau_{1} & =\|\mathbf{p}\| / c .
\end{aligned}
$$

For NLOS paths, the geometrical relationships of the angle parameters of the $m^{\text {th }}$ path are shown in Fig. 4. These relationships can be written as

$$
\begin{aligned}
\theta_{\mathrm{UE}, m} & =\theta_{0}+\tan ^{-1}\left(w_{m, y} / w_{m, z}\right), \\
\phi_{\mathrm{UE}, m} & =-\phi_{0}+\tan ^{-1}\left(w_{m, y} / w_{m, x}\right), \\
\theta_{\mathrm{BS}, m} & =\tan ^{-1}\left(q_{m, y} / q_{m, z}\right), \\
\phi_{\mathrm{BS}, m} & =\tan ^{-1}\left(q_{m, y} / q_{m, x}\right), \\
\tau_{m} & =\left(\left\|\mathbf{q}_{m}\right\|+\left\|\mathbf{w}_{m}\right\|\right) / c .
\end{aligned}
$$

where $\mathbf{w}_{m} \triangleq\left[w_{m, x}, w_{m, y}, w_{m, z}\right]^{\mathrm{T}}=\mathbf{q}_{m}-\mathbf{p}$. Deriving (17) w.r.t. the location parameters and (18) and substituting into $\mathbf{T}$ yields the FIM of the location parameters. Closed-form expressions for LOS PEB and OEB are derived in [13]. The 

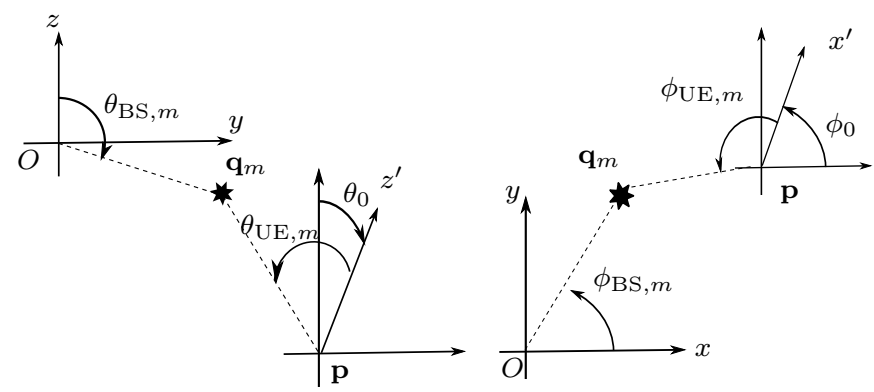

Fig. 4. NLOS relationship between orientation angles and the angles of BS and UE, in the Elevation (left), and Azimuth (right).

full derivations are omitted and instead we provide several key observations.

\section{Key Observations}

From (17) and (18), we note the following.

1) For both LOS and NLOS, the UE position is directly related to $\theta_{\mathrm{BS}, m}, \phi_{\mathrm{BS}, m}$ and $\tau_{m}$. This means that PEB, in addition to being a function of TOA, is a function of DOD in the downlink, and the DOA in the uplink. Since the CRLBs of DOA and DOD are different [13], the PEB in uplink and downlink are not identical.

2) On the other hand, the UE orientation is directly related to $\theta_{\mathrm{BS}, m}, \phi_{\mathrm{BS}, m}, \theta_{\mathrm{UE}, m}, \phi_{\mathrm{UE}, m}$. Therefore, OEB is a function of the DOA and DOD both in the uplink and downlink.

3) In the downlink, beamforming is performed in the BS that has a fixed orientation, and derivatives of the BS angles w.r.t. orientation are zero. Thus, the downlink PEB and OEB are not affected by the UE orientation. On the contrary, the uplink PEB and OEB are sensitive to the UE orientation, where the beamforming is performed. However, only when the BS and UE have the same orientation, uplink and downlink OEB are identical.

\section{Numerical Results and Discussion}

\section{A. Simulation Environment}

1) Geometry: We consider a scenario where a BS with a height of 10 meters and a square array of $N_{\mathrm{BS}}$ antennas is located in the $x z$-plane and centered at the origin. The UE, operating at $f=38 \mathrm{GHz}$, is equipped with a square array of $N_{\mathrm{UE}}$ antennas, and assumed to be tilted by some orientation angle. We investigate the performance over a flat $120^{\circ}$ sector of a sectored cell with a radius of 50 meters. The UE is assumed to be located anywhere within this sector.

2) Transceiver Parameters: We consider an ideal sinc pulse so that $W_{\text {eff }}^{2}=W^{2} / 3$, where $W=125 \mathrm{MHz}, E_{\mathrm{s}} / T_{\mathrm{s}}=0 \mathrm{dBm}$, $N_{0}=-170 \mathrm{dBm} / \mathrm{Hz}$, and $N_{\mathrm{s}}=16$ pilot symbols.

3) Beamforming: We employ directional beamforming, defined in (5). From Fig. 5, in the downlink, the beams directions are chosen such that the beams centers are equi-spaced on the ground. In the uplink, the beams are equispaced on a virtual sector containing the BS and parallel to the UE array. Fig. 6 illustrates the normalized footprint of the beam pattern of the BS (downlink) on the sector. Note that the beam coverage is higher in areas farther away from the BS, in general, since the beam intersection of the sector is ellipse. This is

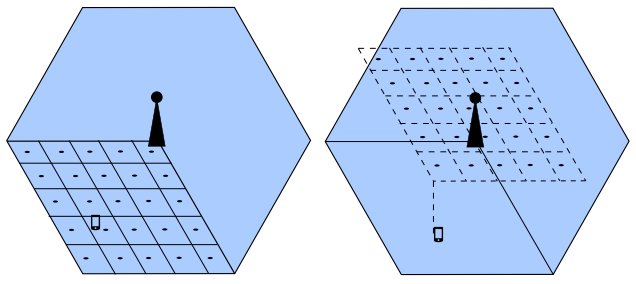

Fig. 5. A cell sectored into three sectors, each served by 25 beams directed towards a grid on the ground in the downlink (left) and towards a virtual grid in uplink (right). The grid has the same orientation as the UE.

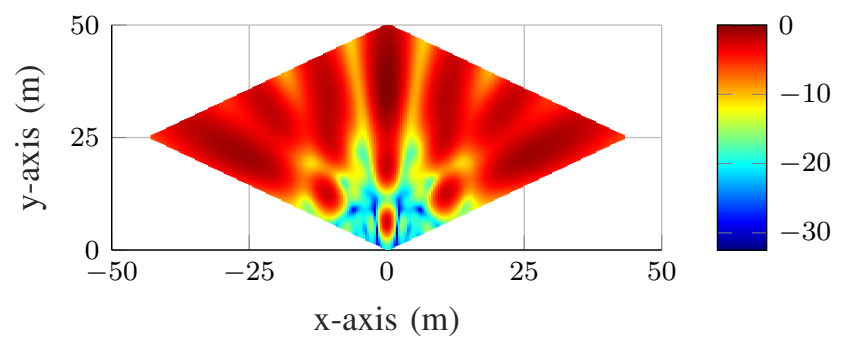

Fig. 6. Normalized footprint (dB) of the 25 beams on the grid in Fig. 5 (left)
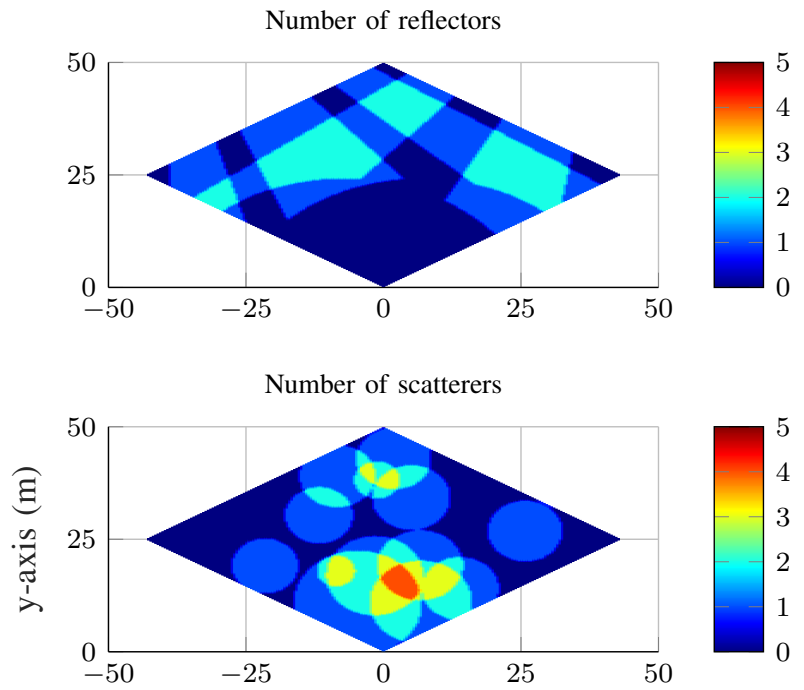

Total number of clusters

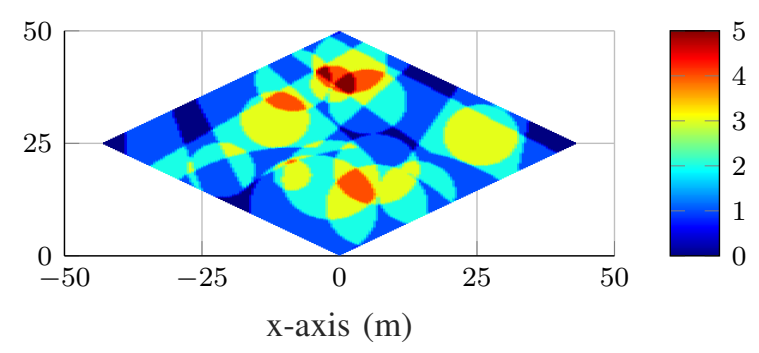

Fig. 7. The number of reflectors (top), clusters (middle), and clusters (bottom) as function of the UE location.

an advantageous feature to combat higher propagation loss at these areas. All the results are obtained with $N_{\mathrm{B}}=25$, $N_{\mathrm{T}}=N_{\mathrm{R}}=144$, unless otherwise stated.

4) Channel: The environment contains scatterers distributed arbitrarily in the 3D space, and reflectors placed close to the sector edge. The number of scatterers and reflectors contributing to the received signal depends on the UE position. For the considered setup, Fig. 7 shows the distribution of the number 

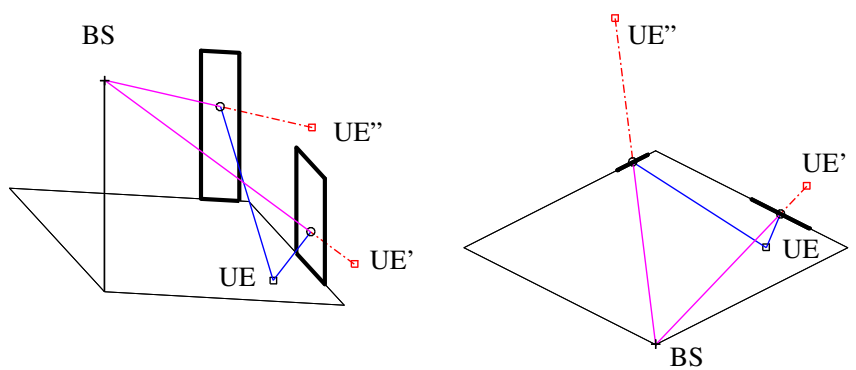

Fig. 8. The virtual transmitter method in 3D (left) and its top view (right). LOS PEB (m)

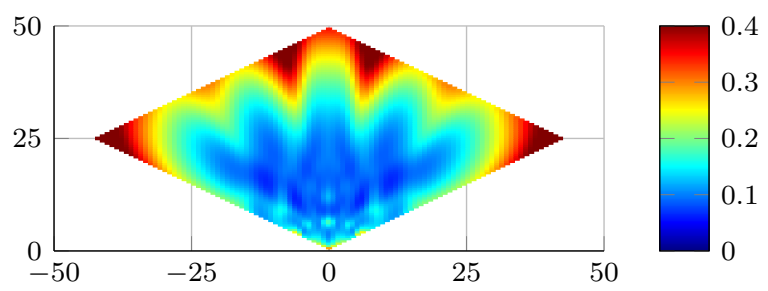

LOS OEB $\left({ }^{\circ}\right)$

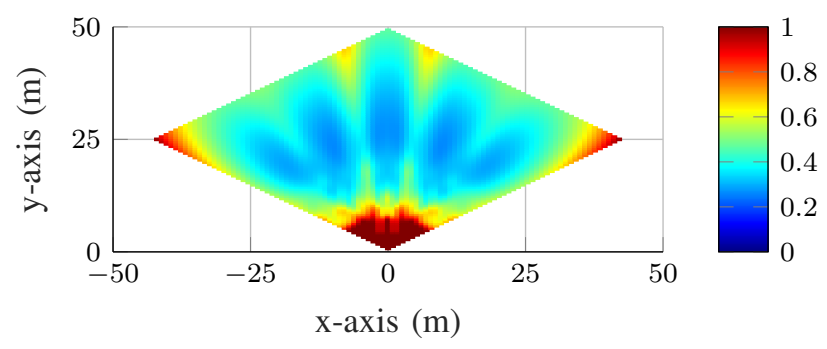

Fig. 9. Downlink LOS PEB and OEB. $N_{\mathrm{BS}}=N_{\mathrm{UE}}=144, N_{\mathrm{B}}=25$.

of clusters (reflectors and scatterers) over the considered sector. Note that a maximum of 5 clusters contribute to the UE signal. In Fig. 7, a cluster is ignored if the received power from that cluster is below $10 \%$ of the LOS.

Accordingly, the complex channel gain of the $m^{\text {th }}$ path is modeled by $\beta_{m}=\left|\beta_{m}\right| e^{j \vartheta_{m}}$ such that

$$
\left|\beta_{m}\right|^{2}=\frac{\lambda^{2}}{(4 \pi)^{2}} \begin{cases}1 / D_{1}^{2} & \text { LOS } \\ \Gamma_{\mathrm{R}} /\left(d_{1, m}+d_{2, m}\right)^{2} & \text { reflector, } \\ \sigma_{\mathrm{RCS}}^{2} /\left(4 \pi\left(d_{1, m} d_{2, m}\right)^{2}\right) & \text { scatterer, }\end{cases}
$$

where $\vartheta_{m}=2 \pi D_{m} / \lambda$, while $\sigma_{\mathrm{RCS}}^{2}=50 \mathrm{~m}^{2}$, and $\Gamma_{\mathrm{R}}=$ 0.7 are the radar cross section, and the reflection coefficient, respectively. To maintain the relationship between angles of incidence and reflection in 3D, we use the virtual transmitter method [16], illustrated in Fig. 8, by which the reflection point is calculated as the intersection point of the reflector and the line connecting the BS to a virtual mirror-image of the UE. It is understood that not all locations in the sector will communicate with the BS via a reflected signal, in which case the reflector is ignored. Finally, note that Fig. 8 provides an illustration of the uplink, but since $d_{2, m}$ and $d_{1, m}$ in (19) are interchangeable, the downlink is the same.

\section{B. Downlink PEB and OEB Under Multipath}

Figs. 9 and 10 show the PEB and OEB for the two cases of LOS and LOS with clusters (LOS + C), respectively. Although incorporating NLOS clusters in the localization does not lower
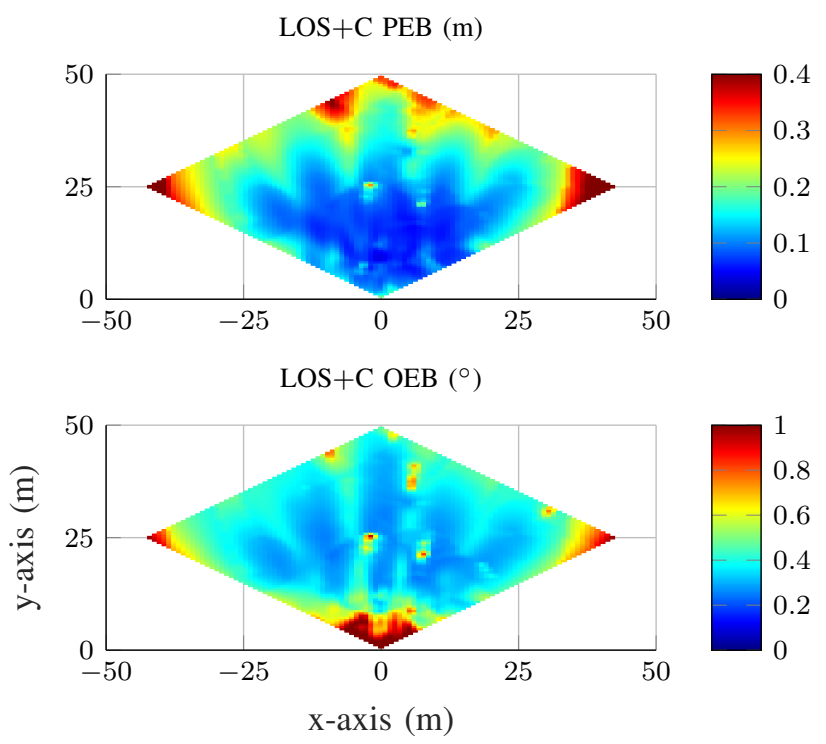

Fig. 10. Downlink LOS + C PEB and OEB. $N_{\mathrm{BS}}=N_{\mathrm{UE}}=144, N_{\mathrm{B}}=25$.

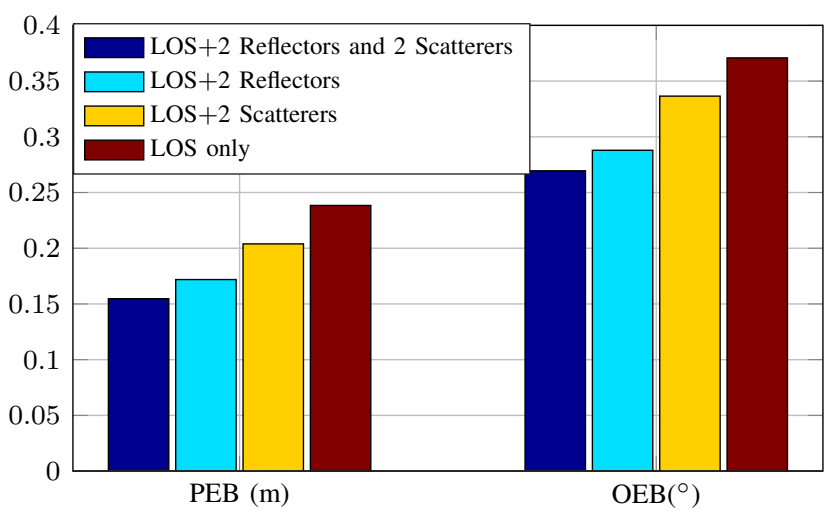

Fig. 11. PEB and OEB over locations with 2 reflectors and 2 scatterers.

the maximum bound value, it does improve the bounds at those locations where the clusters' signal are received. In the illustrated example, the clusters mainly affect the top and center areas of the sector where the map of $\mathrm{LOS}+\mathrm{C}$ has extended green and blue areas, while the red areas shrink. Finally, note the red dots in the central area of the PEB and OEB $(\mathrm{LOS}+\mathrm{C})$. These dots occur because at these locations, the scatterer blocks the LOS path, violating the unique parameters assumption, and causing singularities in the FIM.

To test the effect of reflectors and scatterers separately, we investigate a subset of the locations in Fig. 7, for which 2 scatterer and 2 reflectors contribute to the received signal, as in Fig. 1. We then obtain the average PEB and OEB over these locations for the cases highlighted in Fig. 11. It can be seen that for the considered scenario, on average the PEB and OEB improvement achieved with the reflectors exceed that achieved with the scatterers. This is reasonable since reflectors retransmit most of the incident power directionally, unlike scatterers that retransmits the signal omni-directionally. 


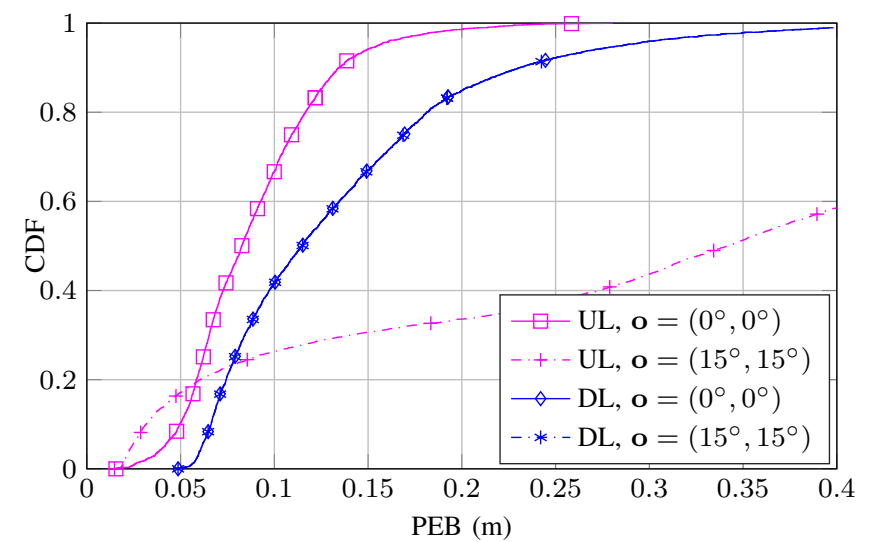

Fig. 12. CDF of the PEB over the entire sector, for uplink and downlink, with different orientation angles.

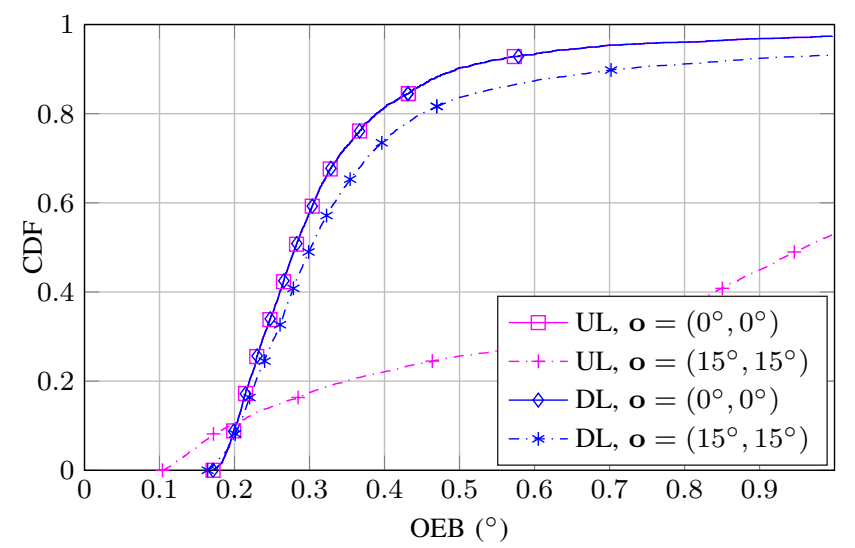

Fig. 13. CDF of the OEB over the entire sector, for uplink and downlink, with different orientation angles.

\section{UE Orientation Impact on PEB and $O E B$}

Considering Fig. 12, the CDF of the PEB is shown for uplink and downlink with two different UE orientation angles. The downlink PEB is a function of the BS angles (DOD), independent of the UE orientation. Therefore, the downlink PEB is identical in both $0^{\circ}$ and $15^{\circ}$ orientation cases. On the contrary, the uplink PEB is highly dependent on the UE orientation, since the beamforming is performed in fixed directions w.r.t. to the UE's frame of reference. As a result, UE beams may miss the BS. With $15^{\circ}$ orientation, this happens more frequently, which degrades the PEB. Finally, although in Fig. 12 the uplink with $0^{\circ}$ orientation is better than the downlink, this is not alway the case. In fact, this depends on the choice of $N_{\mathrm{R}}$, as shown in [13]. In general, downlink is more stable and attains a of $23 \mathrm{~cm} \mathrm{PEB}$, at $90 \% \mathrm{CDF}$.

For the OEB in Fig. 13, the downlink and uplink OEB curves coincide for $0^{\circ}$ yielding similar performance. This is because OEB is a function of DOA and DOD, which are interchangeable when UE and BS have the same orientation. At $90 \% \mathrm{CDF}$, OEB is $0.5^{\circ}$. Finally, when the UE orientation is $15^{\circ}$, OEB is again degraded for both the uplink and downlink.

\section{CONCLUSIONS}

In this paper, we investigated the uplink and downlink PEB and OEB under multipath mmWave propagation and arbitrary array. Based on the considered scenarios, our simulations show that the NLOS clusters improve the localization when a LOS path exists. We observed that, under our model, reflectors improve PEB and OEB, more than scatterers do. Even though uplink localization can offer better localization capabilities than downlink, the former is generally harder since transmit beamforming at UE may point in directions that are not useful for localization.

\section{ACKNOWLEDGMENT}

This work is partly supported by the Australian Research Council's Discovery Projects funding scheme DP140101133, the Spanish R\&D Project TEC2014-53656-R, the EU H2020 projects HIGHTS MG-3.5a-2014-636537 and 5GCAR, and the VINNOVA COPPLAR project, funded under Strategic Vehicle Research and Innovation Grant No. 2015-04849.

\section{REFERENCES}

[1] R. D. Taranto, S. Muppirisetty, R. Raulefs, D. Slock, T. Svensson, and H. Wymeersch, "Location-aware communications for 5G networks: How location information can improve scalability, latency, and robustness of 5G," IEEE Signal Process. Mag, vol. 31, no. 6, pp. 102-112, Nov 2014.

[2] N. Garcia, H. Wymeersch, E. G. Ström, and D. Slock, "Location-aided mm-wave channel estimation for vehicular communication," in IEEE 17th Int. Workshop on Signal Process. Advances in Wireless Commun., July 2016, pp. 1-5.

[3] K. Witrisal, P. Meissner, E. Leitinger, Y. Shen, C. Gustafson, F. Tufvesson, K. Haneda, D. Dardari, A. F. Molisch, A. Conti, and M. Z. Win, "High-accuracy localization for assisted living: 5G systems will turn multipath channels from foe to friend," IEEE Signal Process. Mag, vol. 33, no. 2, pp. 59-70, March 2016.

[4] L. S. Muppirisetty, T. Svensson, and H. Wymeersch, "Spatial wireless channel prediction under location uncertainty," IEEE Trans. on Wireless Commun., vol. 15, no. 2, pp. 1031-1044, Feb 2016.

[5] J. C. Aviles and A. Kouki, "Position-aided mm-wave beam training under NLOS conditions," IEEE Access, vol. 4, pp. 8703-8714, 2016.

[6] N. Akbar, S. Yan, N. Yang, and J. Yuan, "Mitigating pilot contamination through location-aware pilot assignment in massive MIMO networks," in 2016 IEEE Globecom Workshops (GC Wkshps), Dec 2016, pp. 1-6.

[7] S. M. Kay, Fundamentals of Statistical Signal Processing: Estimation Theory. NJ, USA: Prentice-Hall, Inc., 1993.

[8] Y. Shen and M. Z. Win, "Performance of localization and orientation using wideband antenna arrays," in 2007 IEEE International Conference on Ultra-Wideband, Sept 2007, pp. 288-293.

[9] _ , "On the accuracy of localization systems using wideband antenna arrays," IEEE Trans. on Commun., vol. 58, no. 1, pp. 270-280, Jan 2010.

[10] A. Shahmansoori, G. E. Garcia, G. Destino, G. Seco-Granados, and H. Wymeersch, "Position and orientation estimation through millimeter wave MIMO in 5G systems," 2017. [Online]. Available: https://arxiv.org/abs/1702.01605

[11] Y. Han, Y. Shen, X. P. Zhang, M. Z. Win, and H. Meng, "Performance limits and geometric properties of array localization," IEEE Trans. on Inf. Theory, vol. 62, no. 2, pp. 1054-1075, Feb 2016.

[12] A. Guerra, F. Guidi, and D. Dardari, "Single anchor localization and orientation performance limits using massive arrays: MIMO vs. beamforming," 2017. [Online]. Available: https://arxiv.org/abs/1702. 01670

[13] Z. Abu-Shaban, X. Zhou, T. Abhayapala, and H. W. Gonzalo SecoGranados, "Error bounds for uplink and downlink 3D localization in 5G mmwave systems," 2017. [Online]. Available: https://arxiv.org/abs/ 1704.03234

[14] M. Wax and T. Kailath, "Detection of signals by information theoretic criteria," IEEE Transactions on Acoustics, Speech, and Signal Processing, vol. 33, no. 2, pp. 387-392, 1985.

[15] Z. Pi and F. Khan, "An introduction to millimeter-wave mobile broadband systems," IEEE Commun. Mag, vol. 49, no. 6, pp. 101-107, June 2011.

[16] C. Gentner, T. Jost, W. Wang, S. Zhang, A. Dammann, and U. C. Fiebig, "Multipath assisted positioning with simultaneous localization and mapping," IEEE Trans. on Wireless Commun., vol. 15, no. 9, pp. 6104-6117, Sept 2016. 